\title{
INFRARED PHOTOMETRY AND SPECTROSCOPY OF THE BROWN DWARF CANDIDATE PC0025 $+0447^{1}$
}

\author{
JAMES. R. GRAHAM ${ }^{2}$ \\ Astronomy Department, University of California, Berkeley, California 94720, and Palomar Observatory, Caltech, Pasadena, \\ California 91125
}

\author{
K. Matthews, J. L. Greenstein, G. Neugebauer, and C. G. Tinney \\ Palomar Observatory, Caltech, Pasadena, California 91125
}

S. E. PERSSON

Observatories of the Carnegie Institute of Washington, 813 Santa Barbara Street, Pasadena, California 91101

Received 14 April 1992 revised 6 July 1992

\begin{abstract}
We have obtained $I, J, H, K$, and $L^{\prime}$ photometry and infrared spectroscopy $(\lambda / \Delta \lambda \simeq 100)$ from $1.2-2.4$ $\mu \mathrm{m}$ of the unusual emission line M star PC0025 +0447 . The near infrared photometry shows that this star has infrared colors which are comparable to the latest M dwarf stars such as LHS 2924 and LHS 2065. The infrared colors of PCOO25 + 0447 establish that this star is a dwarf and not a distant giant. The infrared spectrum is dominated by strong $\mathrm{H}_{2} \mathrm{O}$ absorption bands, and these bands are the strongest measured in any dwarf. Since $\mathrm{H}_{2} \mathrm{O}$ band strength increases with decreasing temperature it is very likely that PCO025 + 0447 is substantially cooler than any of the stars calibrated by Berriman \& Reid [MNRAS, 227, 315 (1987)], i.e. $T_{\text {eff }}<2450 \mathrm{~K}$. The CO first overtone absorption is much weaker in PC0025 + 0447 than in hotter M dwarfs. Comparison of the spectroscopy of PC0025+0447 with model atmospheres shows correspondence between the observed strength and shape of individual $\mathrm{H}_{2} \mathrm{O}$ bands, although the $\mathrm{H}_{2} \mathrm{O}$ band strength is systematically greater in the models. A practical spectral classification sequence and ultimately a temperature scale based on the strength of the infrared $\mathrm{H}_{2} \mathrm{O}$ bands may be feasible for stars later than M6.5. A preliminary effective temperature for PC0025+0447 based on integrating the observed fluxes yields $T_{\text {eff }}=1900 \pm 600 \mathrm{~K}$. Spectroscopically the band strength suggests the lowest $T_{\text {eff }}$ for any dwarf so far observed. We infer from the observed $T_{\text {eff }}$ and theoretical evolutionary models that PC0025 +0447 is either an old $\left(>10^{9} \mathrm{yr}\right) \mathrm{H}$ burning star, with $\mathscr{M} \simeq 0.08 \mathscr{M}_{\odot}$, or a younger brown dwarf.
\end{abstract}

\section{INTRODUCTION}

The $\mathbf{M}$ dwarf stars are of interest for a variety of reasons. Observations of $\mathbf{M}$ dwarfs are, however, technologically challenging because they are faint, while understanding the evolution and atmospheres of these stars remains at the vanguard of theoretical progress. There also is the possibility that low mass stars are present in sufficient numbers to contribute to the dynamics of the Galactic disk (Bahcall 1986).

PC0025 + 0447 (hereafter abbreviated to PC0025) was discovered by Schneider et al. (1991) in the course of the Palomar CCD grism survey on account of very strong $(E W>250 \AA$ ) $\mathrm{H} \alpha$ emission. Subsequent optical spectroscopy by Greenstein revealed the spectrum of a very red $M$ dwarf with strong $\mathrm{K} \mathrm{I}$, and the strongest known VO bands (Schneider et al. 1991). The weakness of TiO combined with the strength of VO and K I suggest that PC0025 is

'Observations at the Palomar Observatory were made as part of a continuing collaborative agreement between the California Institute of Technology and the Carnegie Institute of Washington.

${ }^{2}$ Alfred P. Sloan Research Fellow. cooler than LHS 2924, continuing the trend to apparently stronger veiling first noted in LHS 2924. PC0025 therefore should be counted among spectroscopic brown dwarf candidates. It has been included in the next set of CCD parallax observations at the USNO, by Monet et al. (1992).

Infrared observations are essential to advance the understanding of $\mathbf{M}$ dwarfs because these stars are infrared objects. For example a dM0 star with $T_{\text {eff }}=3500 \mathrm{~K}$ radiates about $\frac{2}{3}$ of its luminosity at wavelengths longer than 1 $\mu \mathrm{m}$. Therefore, infrared observations are required to establish fundamental properties such as the luminosity and effective temperature.

Berriman \& Reid (1987) have shown that broad-band photometry alone is inadequate to determine $T_{\text {eff }}$ because strong $\mathrm{H}_{2} \mathrm{O}$ absorptions dominate the infrared spectrum, complicating interpretation of $J, H, K$ colors. Thus, it is clear that infrared spectroscopy will play a vital role in determining the nature of low mass stars. The infrared spectra of stars later than dM6.5 are completely unexplored. There is hope that some unexpected diagnostic will appear which will discriminate between H-burning stars and contracting brown dwarfs. At a more fundamental level, one of the largest sources of uncertainty in calculat- 
TABle 1. Photometry. ${ }^{\mathrm{a}}$

\begin{tabular}{lcc}
\hline \hline Filter & Wavelength $(\mu \mathrm{m})$ & Magnitude \\
\hline$I_{C}$ & 0.9 & $19.53 \pm 0.03$ \\
$J$ & 1.25 & $16.11 \pm 0.06$ \\
$H$ & 1.65 & $15.40 \pm 0.02$ \\
$K$ & 2.20 & $14.87 \pm 0.04$ \\
$L^{\prime}$ & 3.7 & $>12.3(3 \sigma)$ \\
\hline
\end{tabular}

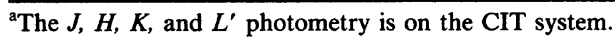

ing the properties of low mass $\left(<0.3 \mathscr{M}_{\odot}\right)$ stars can be traced back to the physics of their atmospheres (Dorman et al. 1989). In low mass stars, which are completely convective, the atmosphere acts as a thermostat which regulates the nuclear energy production in the interior. Hence, infrared spectroscopy, which can be used to check stellar atmosphere models, can also provide an empirical basis for validating theoretical models of the interiors of low mass stars.

\section{OBSERVATIONS AND DATA REDUCTION}

PC0025 was observed at the $f / 70$ Cassegrain focus of the Hale $5 \mathrm{~m}$ telescope with an infrared camera equipped with a Santa Barbara Research Corporation $58 \times 62 \mathrm{InSb}$ direct readout detector array. The pixels correspond to 0 ". 31 on the sky. Aperture photometry, listed in Table 1, in the $J, H, K$, and $L^{\prime}$ bands was derived from images of PC0025. Standard reduction techniques including linearization, sky subtraction, and flat fielding were employed. The magnitude scale was determined from observations of the CIT standards (Elias et al. 1982). Photometry in the $I$ band, on the Cousins system, was also measured at Palomar, using the 60 inch telescope.

The infrared camera is equipped with a transmission grism and slits mechanism so that low resolution spectra can be obtained. Spectra of PC0025 were obtained from 1.16 to $2.46 \mu \mathrm{m}$ using a 1.0 slit and the broad-band photometric filters for order sorting. The effects of terrestrial atmospheric absorption and the instrumental response were corrected for by dividing the observed spectrum of PC0025 by the spectra of early G dwarf stars. This spectral type was chosen from the spectral atlas of Arnaud et al. (1989) on account of the virtually featureless continua of $\mathrm{G}$ dwarfs at this resolution. In particular, the $\mathrm{H}_{2} \mathrm{O}$ and $\mathrm{CO}$ absorption bands in early $\mathbf{G}$ dwarfs are very weak. The reduced spectrum of $\mathrm{PC} 0025$ is shown in Fig. 1.

As the $\mathrm{H}_{2} \mathrm{O}$ bands in the spectra of late type dwarfs such as $\mathrm{PC} 0025$ are of special interest, care was taken to ensure that absorption by terrestrial $\mathrm{H}_{2} \mathrm{O}$ could be corrected for. This was achieved by observing PCOO25 and the spectral standard BS 448 (dG2) at similar air mass; the $K$ spectrum of PC0025 was obtained between air masses of 1.54 and 1.34, while BS 448 was observed at an air mass of 1.56 , directly after PC0025. To check for systematic errors in correcting for atmospheric absorption, which might lead to the depressions in flux close to the edges of the atmospheric windows, we observed the dG1 star BS 5618 at high air

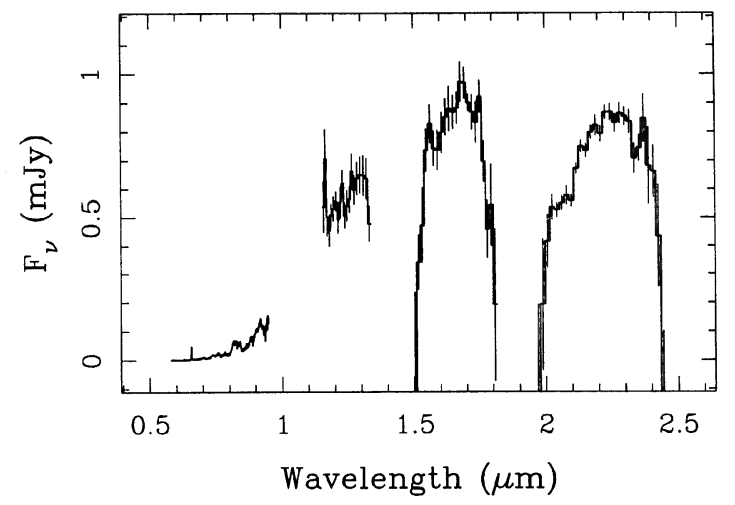

FIG. 1. The optical infrared spectrum of PCO025+0447. The spectrum is dominated by very strong absorption in the wings of the 1.4, 1.9, and $2.7 \mu \mathrm{m} \mathrm{H}_{2} \mathrm{O}$ bands. The optical data are taken from Schneider et al. (1991).

mass (2.4) before PC0025. The ratio of the observed fluxes of BS 5618 at air mass 2.4 and BS 448 at air mass 1.6 is flat and featureless from 1.95 to $2.47 \mu \mathrm{m}$ with the exception of the two terrestrial $\mathrm{CO}_{2}$ bands at 2.02 and $2.06 \mu \mathrm{m}$. The stronger band at $2.02 \mu \mathrm{m}$ yields an absorption of $0.4 \mathrm{mag}$ per air mass. Since the standard was observed at higher airmass than PCO025 we will overestimate the flux from PC0025 at $2.02 \mu \mathrm{m}$ by $\simeq 6 \%$ and by $\simeq 2 \%$ at $2.06 \mu \mathrm{m}$. Consequently, we are confident that the atmospheric absorption correction is accurate and that the strong absorptions seen in Fig. 1 are intrinsic. The airmass differences between object and standard for the $H$ and $J$ spectra were -0.32 and 0.03 , respectively; small enough that the atmospheric correction is accurate to better than a few percent throughout the observed wavelength range.

The ratioed spectra of $\mathrm{PCOO25}$ were converted to a flux density scale by assuming that the spectrum of the $G$ dwarfs could be described by a blackbody at a temperature equal to the effective temperature. The grism spectra are not photometric on account of the narrow slit used to obtain these data. Therefore, we used the broad-band photometry to establish the flux calibration. This was achieved by convolving the reduced spectra with the wavelength response tracings of the $J, H$, and $K$ filters installed in the dewar, and demanding that the integrated fluxes match the broad-band photometry. The magnitudes were converted to flux densities using stellar models and absolute visual measurements of $\alpha$ Lyr (Oke \& Schild 1970).

\section{INFRARED PHOTOMETRY}

The optical and infrared colors of late $\mathbf{M}$ dwarfs are invaluable for establishing the physical properties of these stars. Infrared $J-H$ and $H-K$ colors are useful because they are sensitive to the temperature and the nature of the opacity sources (Mould 1976). They therefore can be used to discriminate between giants and dwarfs and between dwarfs of different chemical abundances. The position of PC0025 on the $J-H, H-K$ color plane is shown in Fig. 2. 


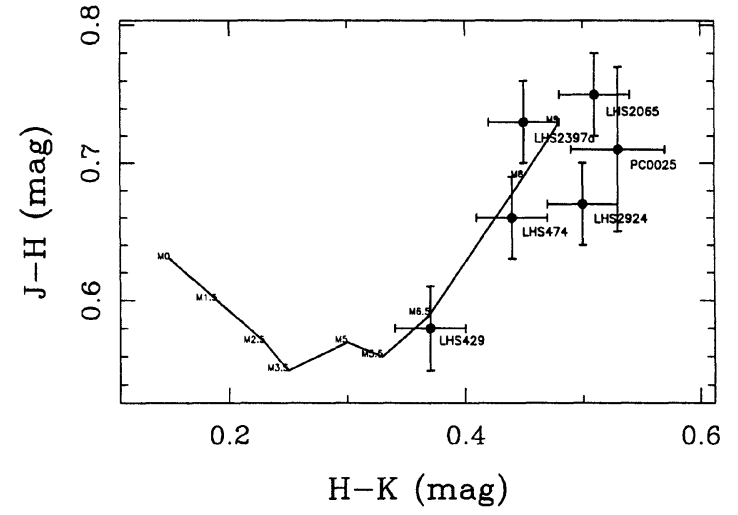

FIG. 2. The $J-H-H-K$ diagram showing the position of PCOO25 in relation to other late dwarfs. The solid line shows the loci of dwarfs from Leggett (1992).

Also shown on this diagram is the dwarf sequence from Leggett (1992), and the location of individual $\mathbf{M}$ dwarfs later than dM6. Late-type giants and dwarfs occupy distinct regions of the $J-H-H-K$ diagram. For a given $H-K$, giants are $\simeq 0.3 \mathrm{mag}$ redder in $J-H$. Thus the infrared colors of PCOO25 demonstrate that PCOO25 is a dwarf star and not a giant, in accord with Schneider et al.'s analysis of the strength of K I in the optical spectrum. PCO025 lies at the red end of the locus of $M$ dwarf colors. The infrared colors of PCO025 and the dwarfs-LHS 2924 (dM9e) and LHS 2065 (dM9e)-(Kirkpatrick et al. 1991) are comparable. These three stars have similar colors, and are significantly redder in $J-H-H-K$ colors than the dM8's.

Optical-infrared colors such as $V-K$ provide precise color temperatures (Bessell 1991) and the basis of accurate color-luminosity relations (Greenstein 1989) on account of the long wavelength baseline. Schneider et al. (1991) used preliminary infrared photometry and the $M_{\text {bol }}$ vs $V-K$ and $I-K$ relations to conclude that $M_{\text {bol }}>13.7 \mathrm{mag}$. Since the dominant sources of uncertainty continues to be in the extrapolation of the color luminosity relation, the new infrared results do not alter this conclusion.

The optical-infrared color of $V-K=9.1 \pm 0.4 \mathrm{mag}$ also places PC0025 among the dM9 stars. An extrapolation of the $T_{\text {eff }}$ vs $V-K$ relation, using the calibration of Berriman $\&$ Reid (1987) for earlier stars ( $\leqslant \mathrm{dM} 6.5$ and $T_{\text {eff }} \geqslant 2450$ K), suggests $T_{\text {eff }} \simeq 2000 \pm 100 \mathrm{~K}$ for PC0025. Further discussion of the optical-infrared colors of PCOO25 is limited by the accuracy of the optical data.

\section{THE INFRARED SPECTRUM}

The most immediate result of the spectroscopic data comes from the $2 \mu \mathrm{m}$ observations of PC0025, which show strong absorption at $2.0 \mu \mathrm{m}$ and only a relatively weak depression in the flux at $2.3 \mu \mathrm{m}$. Comparison of PC0025 with the stars in the the $2 \mu \mathrm{m}$ spectral atlas of Arnaud et al. (1989) clearly confirms that these are characteristics of late dwarfs. PCOO25 lacks the strong $\mathrm{CO}$ absorption at $2.3 \mu \mathrm{m}$ which would identify it as a giant at intergalactic distance.

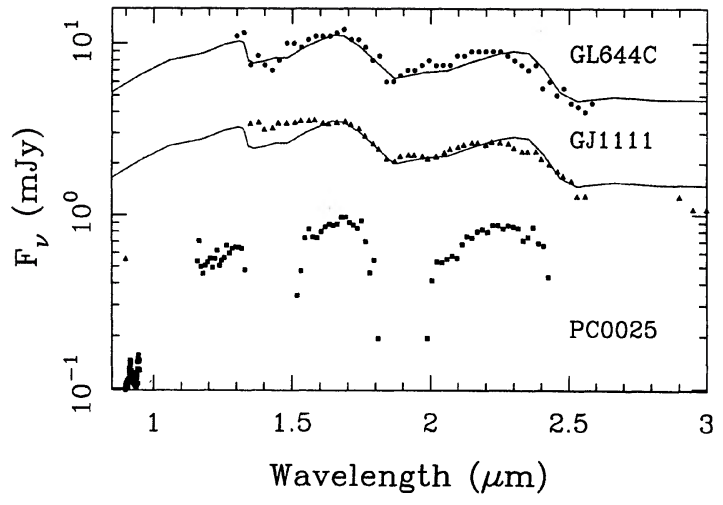

FIG. 3. The optical-infrared spectrum of PC0025 (squares) compared with the late dwarf GJ1111 (triangles) (dG6.5, $T_{\text {eff }}=2450$ $\mathrm{K})$, GL644C (circles) (dG7, $\left.T_{\text {eff }}=2450 \mathrm{~K}\right)$, and a $3000 \mathrm{~K}$, $\log (g)=4.75$ model atmosphere (line) (Mould 1976). This figure emphasizes the strength of the $\mathrm{H}_{2} \mathrm{O}$ bands at $1.4,1.9$, and $2.7 \mu \mathrm{m}$ in PCO025 relative to earlier stars.

Figure 3 permits comparison of the optical-infrared spectrum of PC0025 with observations of GJ 1111 (dM6.5 $\left.T_{\text {eff }}=2450 \mathrm{~K}\right)$ and GL $644 \mathrm{C}\left(\mathrm{dM} 7 T_{\text {eff }}=2450 \mathrm{~K}\right)$ from Berriman \& Reid (1987) and a $M$ dwarf model atmosphere with $T_{\text {eff }}=3000 \mathrm{~K}, \log g=4.74$ and solar (old disk) abundances from Mould (1976). GJ 1111 and GL 644C are the latest $\mathbf{M}$ dwarfs for which infrared spectroscopic observations are available in the literature. GJ 1111 is a young disk star, GL $644 \mathrm{C}$ belongs to the old disk.

Two aspects of the spectrum of PCOO25 become apparent from this comparison: (1) the spectral energy distribution is very red; (2) the absorption bands at the edges of the terrestrial atmospheric windows are exceptionally strong. These absorption features are qualitatively similar to those seen in hotter $\mathbf{M}$ dwarfs and are most likely due to the wings of the $1.4,1.9$, and $2.7 \mu \mathrm{m} \mathrm{H}_{2} \mathrm{O}$ bands. Although absorption by terrestrial $\mathrm{H}_{2} \mathrm{O}$ hampers observations at 1.4 and $1.9 \mu \mathrm{m}$, at the temperatures appropriate to stellar atmospheres of dwarfs, the red wings of these bands extend well into the wavelength range accessible from the ground. The absorptions in the red wings of the 1.4 and $1.9 \mu \mathrm{m}$ $\mathrm{H}_{2} \mathrm{O}$ bands are substantially stronger in $\mathrm{PCOO25}$ than in the comparison stars, but there is no need to invoke an additional opacity source at these wavelengths. There is a characteristic shoulder or step in the $\mathrm{H}_{2} \mathrm{O}$ opacity at 2.1 $\mu \mathrm{m}$ (Auman 1967) which is responsible for the distinct depression in the flux at this wavelength in the model atmosphere. Such a step is clearly visible in the $2 \mu \mathrm{m}$ spectrum of PCOO25. The strength of the observed $2.1 \mu \mathrm{m} \mathrm{H}_{2} \mathrm{O}$ step is correspondingly stronger in PCO025 than in GJ 1111 or GL $644 C$. Consequently, it seems reasonable to attribute the unusually strong absorption bordering the peaks of the $H$ and $K$ spectra to intense $\mathrm{H}_{2} \mathrm{O}$ absorption.

The strength of the red wing of the $1.9 \mu \mathrm{m} \mathrm{H}_{2} \mathrm{O}$ band has been quantified using an $\mathrm{H}_{2} \mathrm{O}$ index defined as the color difference of magnitudes measured in narrow bands within the $K$ window. We have adopted the system of Persson et al. (1977) which defines $\left[\mathrm{H}_{2} \mathrm{O}\right]=[2.0]-[2.2]$. The $\mathrm{H}_{2} \mathrm{O}$ index was calculated from the spectrum of PCOO25 using 
the filter band passes described by Persson et al. and assuming that the zero point of the magnitude system is defined by $\alpha$ Lyrae with a color temperature of $10000 \mathrm{~K}$. Using this system, $\left[\mathrm{H}_{2} \mathrm{O}\right]=0.67 \pm 0.06 \mathrm{mag}$ for $\mathrm{PCO} 25$, which is, by a substantial margin, the largest measured value of the $\mathrm{H}_{2} \mathrm{O}$ index. For comparison, $\left[\mathrm{H}_{2} \mathrm{O}\right]=0.33 \mathrm{mag}$ for GL $406\left(T_{\text {eff }}=2600 \mathrm{~K}\right)$, which has one of the largest previously measured $\mathrm{H}_{2} \mathrm{O}$ indices.

A number of factors may account for the the strength of the $\mathrm{H}_{2} \mathrm{O}$ bands in PC0025: principally effective temperature and chemical abundance. Infrared spectroscopy, narrow-band filter photometry, and model atmospheres of $\mathrm{M}$ dwarfs show a marked strengthening of the $\mathrm{H}_{2} \mathrm{O}$ bands with decreasing effective temperature (Baldwin et al. 1973; Mould 1976; Persson et al. 1977; Arnaud et al. 1989; Allard 1990). From early $M$-type stars $\left(T_{\text {eff }} \simeq 3500 \mathrm{~K}\right)$, where $\mathrm{H}_{2} \mathrm{O}$ bands first become prominent, their strength increases steeply all the way to the coolest known $M$ stars $\left(T_{\text {eff }} \simeq 2500 \mathrm{~K}\right)$. The model atmospheres of Allard, which she kindly made available in tabular form, predict that this trend continues from 2500 to $2000 \mathrm{~K}$. There is little variation of the $\mathrm{H}_{2} \mathrm{O}$ band strength with the range of surface gravities appropriate for dwarfs (Mould 1976).

The strength of the $\mathrm{H}_{2} \mathrm{O}$ bands are sensitive to chemical abundances. Model atmospheres predict a slow weakening as the total metal abundance, relative to $\mathrm{H}$, decreases (Mould 1976; Allard 1990). Bessell \& Scholz (1989), Bessell (1991), and Leggett (1992) demonstrate a dependence of color on metallicity, which they attribute to an $\mathrm{H}_{2} \mathrm{O}$ band strength which increases with decreasing metallicity. Clearly, the dependence of $\mathrm{H}_{2} \mathrm{O}$ opacity on metallicity needs to be established. The comparison stars shown in Fig. 3, GL 644C and GJ 1111, belong to old disk and young disk kinematic populations, but have the same temperature, and therefore differences may be attributed to distinct chemical abundances. The spectra of these stars are essentially identical, suggesting that the effect of metallicity is small -at least compared to temperature. The 1.4 $\mu \mathrm{m} \mathrm{H}_{2} \mathrm{O}$ band may be stronger in GL $644 \mathrm{C}$, but note that the signal to noise for GL $644 \mathrm{C}$ is lower than for GJ 1111 , especially at this wavelength. There is no evidence from the optical spectrum of PCOO25 for unusual abundances. Furthermore, the recent Monet et al. (1992) survey shows no very red metal-poor subdwarfs exist. Thus, we conclude that the temperature effect is the dominant one at work in PC0025, and the great strength of the $\mathrm{H}_{2} \mathrm{O}$ absorption in PCO025 indicates that this star is substantially cooler than the latest $\mathrm{M}$ dwarfs for which $\left[\mathrm{H}_{2} \mathrm{O}\right]$ index has been measured, i.e., $T_{\text {eff }}<2500 \mathrm{~K}$.

Unless some unexpected new continuum opacity source, e.g., dust grains, turns up, $\mathrm{H}_{2} \mathrm{O}$ bands will become increasingly prominent below $T_{\text {eff }}=2500 \mathrm{~K}$. We take as prima facie evidence for this statement the model atmospheres kindly provided by Allard (1990). Model atmosphere calculations for late-type dwarfs are complex, beset with uncertainties concerning their structure and opacity sources. Therefore it is valuable to show that increasing $\mathrm{H}_{2} \mathrm{O}$ strength is not an unexpected result. The depth of a molecular absorption depends on both the ratio of band-to- continuum opacity and on the temperature gradient (governing the limb darkening). Both these factors favor increasing $\mathrm{H}_{2} \mathrm{O}$ band strength because: (1) as the temperature decreases from 4000 to $1700 \mathrm{~K}$ the $\mathrm{H}_{2} \mathrm{O}$ opacity becomes larger relative to the continuum opacity owing to the rapid increase of $\mathrm{H}_{2} \mathrm{O}$ abundance relative to $\mathrm{H}^{-}$and $\mathrm{H}_{2}^{-}$- the dominant sources of continuum absorption (Auman 1967; Mould 1976); (2) so far, no suggestion has surfaced as to how one might reduce the temperature contrast, to make the atmosphere of dwarfs cooler than $T_{\text {eff }}$ $=2500 \mathrm{~K}$ more nearly isothermal.

\section{THE EFFECTIVE TEMPERATURE}

Berriman \& Reid (1989) have derived effective temperatures for their sample of $\mathbf{M}$ dwarfs by determining the integrated flux from their observations and finding the temperature of a blackbody, normalized to the flux at $2.2 \mu \mathrm{m}$, which gives the same integrand. This method should give a satisfactory answer if line blanketing or backwarming at $2.2 \mu \mathrm{m}$ is insignificant. Mould's (1976) models suggested that this assumption will not introduce serious errors for stars with $T_{\text {eff }} \simeq 3000 \mathrm{~K}$.

The colors and spectrum of PC0025 suggest that $T_{\text {eff }}$ $<2500 \mathrm{~K}$. Allard's (1990) models indicate that at such low temperature significant backwarming is present at 2.2 $\mu \mathrm{m}$, and therefore Berriman and Reid's technique cannot be used. Instead we use a semiempirical variant of their method based on Allard's model atmospheres. The model atmospheres provide two factors-the degree of backwarming at $2.2 \mu \mathrm{m}$ and the amount of flux which is radiated longward of $2.4 \mu \mathrm{m}$. The models are not very accurate (cf. Sec. 6), but these are relatively small corrections which do not vary strongly with temperature in the range of interest. Between $T_{\text {eff }}=2000$ and $2500 \mathrm{~K}$ backwarming increases the flux at $2.2 \mu \mathrm{m}$ by $17 \%-13 \%$, respectively. Out-of-band radiation amounts to $18 \%-15 \%$ in the same temperature range. To calculate the integrated flux shortward of $2.4 \mu \mathrm{m}$ we assume that the gaps in the spectrum are bridged using linear interpolation. The corrected integrated flux is $1.3 \pm 0.6 \times 10^{-12} \mathrm{erg} \mathrm{s}^{-1} \mathrm{~cm}^{-2}$ and corresponds to $M_{\text {bol }}=14.3 \pm 0.5-5 \log _{10}(d / 60 \mathrm{pc}) \mathrm{mag}$. The flux density at $2.2 \mu \mathrm{m}$, corrected for backwarming, fixes the solid angle subtended by the star for a given temperature blackbody-i.e., comparison of the calculated and observed flux determines the angular diameter of the star. We then use this solid angle to find the temperature of a blackbody that gives the observed integrated flux. Using the $2000 \mathrm{~K}$ model atmosphere leads to $T_{\text {eff }}=1900 \pm 600 \mathrm{~K}$. The correction factors for the $2500 \mathrm{~K}$ model give a temperature which is $160 \mathrm{~K}$ higher. Thus, the uncertainties are dominated by statistical errors. The large range allowed range in $T_{\text {eff }}$ may be somewhat surprising, since it corresponds to a factor of 13 uncertainty in total surface flux $\left(\sigma T^{4} / \pi\right)$. In this example the monochromatic flux density is measured at a wavelength close to the blackbody maximum, so that the solid angle is very sensitive to temperature. The method works best when the normalizing flux lies on the Rayleigh-Jeans part of the spectrum where the 
flux is proportional to $T$, and not a higher power. Therefore, spectrophotometry extending to $\lambda \gtrsim 5 \mu \mathrm{m}$ will be necessary for accurate $T_{\text {eff }}$ determinations for very cool dwarfs. The weakness of the Berriman and Reid method is that none of the information on the shape of the spectrum is used. Reliable model atmospheres, which are not currently available, are required to use that information.

\section{COMPARISON WITH MODEL ATMOSPHERES}

Figure 3 shows that the $T_{\text {eff }}=3000 \mathrm{~K}$ model of Mould (1976) provides a poor fit to the observations of PCO025. Figure 4 shows the observations of PCO025 together with two models of Allard (1990) with $T_{\text {eff }}=2500 \mathrm{~K}$ and $T_{\text {eff }}$ $=2000 \mathrm{~K}$. Both models have been normalized to the flux density of PCO025 at $2.2 \mu \mathrm{m}$. The $2500 \mathrm{~K}$ model reproduces the depth of the $\mathrm{H}_{2} \mathrm{O}$ bands, but the $\mathrm{H}_{2} \mathrm{O}$ bands in the $2000 \mathrm{~K}$ model are too strong. Although the $2500 \mathrm{~K}$ model gets the absorption strength right, it fails to reproduce the overall energy distribution. The disagreement is especially bad at $1.2 \mu \mathrm{m}$. The latter result is not entirely unexpected since the agreement between observed infrared broad-band colors and model atmospheres for hotter $\mathbf{M}$ dwarfs is also unsatisfactory (Persson et al. 1977). Allard (1992) suggests that the strong absorption between 1.2 and $1.32 \mu \mathrm{m}$ is due to the $0-1$ and $1-2$ bands of $\mathrm{FeH}$, which are not included in the calculation.

Another aspect of the infrared spectrum of $\mathrm{PCOO25}$ highlighted by Fig. 4 is the weakness of $\mathrm{CO}$. The position of the $R$ branch $v=0-2$ band head at $2.294 \mu \mathrm{m}$ is clearly indicated by Allard's model atmosphere. On the other hand CO is present in GJ 1111 \& GL $644 C$ (Fig. 3). The absence of $\mathrm{CO}$ absorption may be evidence for a low abundance of this molecule, either because the $\mathrm{C}$ abundance is low, or because $\mathbf{C}$ is locked up in graphite grains.

There is a systematic discrepancy between the strength of the $\mathrm{H}_{2} \mathrm{O}$ bands in stars and that predicted by Mould's (1976) and Allard's (1990) model atmospheres. For example, consider GJ 1111 ( $T_{\text {eff }}=2450 \mathrm{~K}$ ), which is shown in Fig. 3. The $3000 \mathrm{~K}$ model provides a fair fit to the strength of the $\mathrm{H}_{2} \mathrm{O}$, but the model at the correct temperature for this star $(2500 \mathrm{~K})$ clearly has too much $\mathrm{H}_{2} \mathrm{O}$ absorption to match the observations. From our estimate of $T_{\text {eff }}$ for PC0025 we might have expected the $2000 \mathrm{~K}$ model to be appropriate. However, once again a hotter model is in closer accord with the observed depth of the $\mathrm{H}_{2} \mathrm{O}$. The observed weakness of $\mathrm{H}_{2} \mathrm{O}$ bands relative to the model predictions might indicate the presence of an additional continuum opacity source. The observations of Berriman \& Reid (1987) beyond $2.2 \mu \mathrm{m}$ rule out warm dust as this source from the absence of substantial thermal reradiation in hotter stars. However, if dust is restricted to the photospheric layers and is in equilibrium at the photospheric temperature it will not be revealed by a long wavelength excess. The uncertainties in the temperature of PC0025 are large enough that its atmosphere may be sufficiently cool for gas and refractory grains to coexist.

While there are clearly problems with the currently available model atmospheres, the qualitative agreement be-

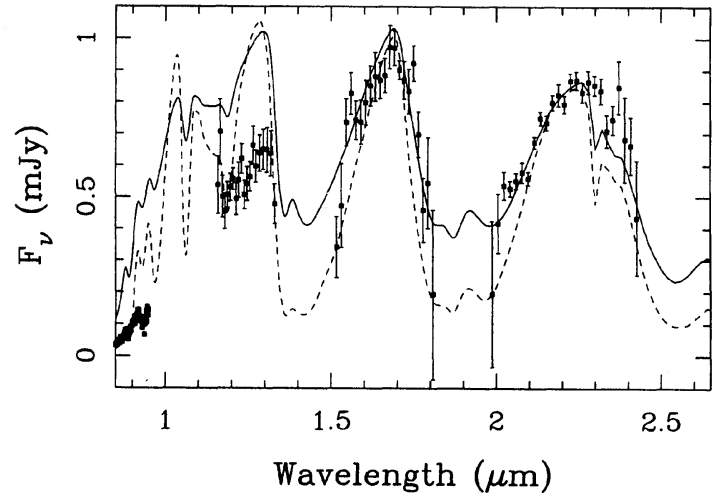

FIG. 4. Observations of PCOO25 together with two models of Allard (1990) with $T_{\text {eff }}=2500 \mathrm{~K}$ (solid) and $T_{\text {eff }}=2000 \mathrm{~K}$ (dashed). Both models have been normalized to the flux of PCO025 at $2.2 \mu \mathrm{m}$. The hotter model reproduces the depth of the $\mathrm{H}_{2} \mathrm{O}$ bands, but fails to reproduce the overall energy distribution. Note the weakness of the CO $v=0-2$ band head at $2.3 \mu \mathrm{m}$.

tween observations and theory begins to be encouraging. The data presented here suggest that it might be possible to construct a practical temperature scale based on the strength of $\mathrm{H}_{2} \mathrm{O}$ bands from dM6.5 to dM9 and perhaps beyond. For there to be a simple mapping from $\mathrm{H}_{2} \mathrm{O}$ band strength to temperature, the effects of metallicity must be understood. This is one of the issues that will be resolved by future infrared spectroscopic studies of late dwarfs.

\section{THE NATURE OF PC0025+0447}

The key question about the late $\mathbf{M}$ dwarfs which we hope infrared observations will answer is: are these stars above or below the main sequence $\mathrm{H}$ burning limit? This is a difficult question to answer because the luminosity and temperature of low mass stars depend on mass and age. As a consequence, a young brown dwarf can masquerade as an older $\mathrm{H}$ burning star. Therefore, it will be difficult to come to any definitive conclusions regarding the status of PCO025 with the limited information we have available.

Figure 5 shows contours of $T_{\text {eff }}$ on the mass-age plane for model B of Burrows et al. (1989). We have plotted the contour for the measured value of $T_{\text {eff }}$ together with the $1 \sigma$ limits. Thus Fig. 5 shows the allowed region in mass-age parameter space where $\mathrm{PCOO25}$ may exist. If the mass limit for $\mathrm{H}$ burning stars is $0.08 \mathscr{M}_{\odot}$, then PC0025 is a brown dwarf if $t<10^{9} \mathrm{yr}$. The age of PC0025 is unknown because kinematic data, which would allow this star to be assigned to a specific population, are unavailable. If the chromospheric activity of PCOO25, as evidenced by strong $\mathrm{H} \alpha$ emission, is taken to imply youth, i.e., belonging to the young disk, then $t \lesssim 7 \times 10^{8} \mathrm{yr}$. At this age the allowed region in Fig. 5 excludes $H$ burning stars at the $1 \sigma$ level. The link between chromospheric activity and youth is not unique; several old disk stars have $\mathrm{H} \alpha$ emission. The presence of chromospheric activity in older stars is usually associated with the presence of a nearby companion (e.g., Giampapa \& Liebert 1986). The optical or infrared images 


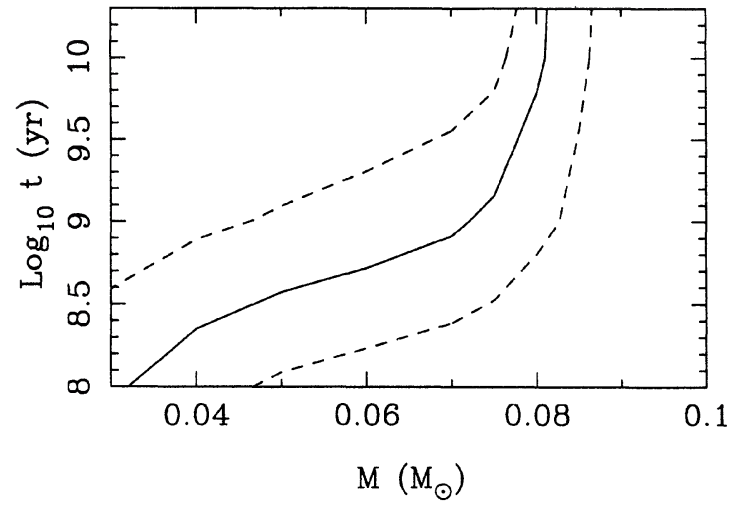

FIG. 5. The contours for the measured value of $T_{\text {eff }}$ (solid) together with the $1 \sigma$ limits (dashed) on the mass-age plane according to the evolutionary models of Burrows et al. (1989). The area between the dashed contours shows the allowed region in massage parameter space where PCO025 may exist. PCO025 is either an old ( $>10^{9}$ yr) $\mathrm{H}$ burning star, with $\mathscr{M} \simeq 0.08 \mathscr{M} \odot$, or a younger brown dwarf.

of PC0025 show no evidence that it is part of a binary system and therefore PCOO25 may be a young object. Despite the evidence for an active chromosphere from the large equivalent width $\mathrm{H} \alpha, \mathrm{PCO} 25$ was not detected in a one-hour Very Large Array integration which achieved $1 \sigma$ $=0.038 \mathrm{mJy}$ at $8.4 \mathrm{GHz}$ (Goss 1992). On the other hand, PCO025 may be old. There is no association with a nearby region of ongoing star formation. The weakness of the 2.3 $\mu \mathrm{m}$ CO feature might indicate that $\mathrm{PC} 0025$ is an old, metal-poor, star. Even if other evidence becomes available which indicates the age of PCOO25 then the errors in the effective temperature determination will still have to be significantly improved before we can say with any confidence that this star is a genuine brown dwarf.

\section{CONCLUSIONS}

Infrared photometry and spectroscopy of PC0025 have demonstrated that this star is a cool, $T_{\text {eff }} \simeq 2000 \mathrm{~K}$ dwarf. The $\mathrm{H}_{2} \mathrm{O}$ absorption bands at 1.4 and $1.9 \mu \mathrm{m}$ are exceptionally prominent and are stronger than previously measured in any dwarf. We conclude that $\mathrm{H}_{2} \mathrm{O}$ band strength increases dramatically as the effective temperature decreases from 3000 to $2000 \mathrm{~K}$. Therefore it may be practical to establish an infrared spectroscopic classification sequence based on the strength of the $\mathrm{H}_{2} \mathrm{O}$ bands.

PC0025 may be a H-burning star or a substellar object. The status of PCOO25 will remain uncertain until the parallax is known, the kinematic population is determined, and the accuracy of $T_{\text {eff }}$ is improved.

We are indebted to France Allard for making her $\mathbf{M}$ dwarf model atmospheres available to us, and for invaluable assistance in interpreting the infrared spectra of PC0025. This work was supported in part by NSF and the Alfred P. Sloan Foundation.

\section{REFERENCES}

Allard, F. 1990, thesis, Ruprecht-Karls-Universtät, Heidelberg

Allard, F. 1992, private communication

Arnaud, K. A. Gilmore, \& G. Cameron, A. C. 1989, MNRAS, 237, 495

Auman, J. R. 1967, ApJS, 14, 171

Bahcall, J. N. 1986, ARA\&A, 24, 577

Baldwin, J. R., Frogel, J. A., \& Persson, S. E. 1973, ApJ, 184, 427

Berriman, G., \& Reid, N. 1987, MNRAS, 227, 315

Bessell, M. S. 1991, AJ, 101, 662

Bessell, M. S., \& Scholz, M. 1989, Accuracy of Elemental Abundances

from Stellar Atmospheres: Springer Lecture Notes in Physics, edited by R. Wehrse (Springer, Berlin)

Burrows, A., Hubbard, W. B., \& Lunine, J. I. 1989, ApJ, 345, 939

Dorman, B., Nelson, L. A., \& Chau, Y. 1989, ApJ, 342, 1003
Elias, J. H., Frogel, J. A., Matthews, K., \& Neugebauer, G. 1982, AJ, 87, 1031

Giampapa, M. S., \& Liebert, J. 1986, ApJ, 305, 784

Goss. W. M. 1992, private communication

Greenstein, J. L. 1989, PASP, 101, 787

Kirkpatrick, J. D., Henry, T. J., \& McCarthy, D. W. 1991, ApJS, 77, 417

Leggett, S. K. 1992, ApJS (in press)

Monet, D. G. Dahn, C. C. Vrba, F. J. Harros, H. C. Pier, J. R., Luginbuhl, C. B., \& Ables, H. D. 1992, AJ, 103, 638

Mould, J. R. 1976, A\&A, 48, 443

Oke, J. B., \& Shild, R. E. 1970, ApJ, 161, 1015

Persson, S. E., Aaronson, M., \& Frogel, J. A. 1977, AJ, 82, 729

Schneider, D. P., Greenstein, J. L., Schmidt, M., \& Gunn, J. E. 1991, AJ, 102,1180 